\title{
Enfrentamento do paciente oncológico frente quimioterapia: contribuições da enfermagem
}

\author{
Facing the oncological patient in front of chemotherapy: nursing contributions
}

Frente al paciente oncológico quimioterapia frontal: contribuciones de enfermería

\section{Resumo}

O câncer ocorre quando o aumento das células do corpo estão fora de controle e por consequência a quimioterapia é um dos tratamentos mais utilizados para o domínio sistêmico da doença, uma vez que essa alternativa está sendo muito relevante no combate à doença de forma singularizada ou combinada, porém, é capaz de causar no indivíduo fragilidade física e emocional de forma vultosa. Trata-se de uma pesquisa bibliográfica de abordagem qualitativa, com o objetivo de descrever o enfrentamento do paciente oncológico. Após a associação de todos os descritores, foram encontrados 151.280 artigos, excluídos 151.266 e selecionados 14. Perante as contribuições da enfermagem, é necessário identificar as fundamentais particularidades de cada paciente, para uma contribuição de modo integral e resultados terapêuticos eficientes. Posterior à leitura reflexiva emergiram duas categorias: Contribuições da enfermagem no enfrentamento do paciente oncológico diante da quimioterapia e Ótica do paciente oncológico frente a quimioterapia. A enfermagem tem papel fundamental diante dos paradigmas que permeiam o enfrentamento do indivíduo acometido pela doença oncológica, visto que na maioria dos casos o doente necessita primordialmente de apoio assistencial. Conclui-se que é de extrema relevância que se avalie os principais fatores que podem contribuir para o sofrimento do paciente oncológico diante do tratamento à base de quimioterápicos, para um melhor desempenho profissional e qualidade da assistência prestada ao doente.

Palavras-chave: Enfermagem; Quimioterapia; Oncologia. 


\begin{abstract}
Cancer occurs when the increase in body cells is out of control and consequently chemotherapy is one of the most used treatments for the systemic domain of the disease, since this alternative is becoming increasingly relevant in combating the disease in a unique way or combined, however, it is capable of causing the individual physical and emotional weakness in a great way. This is a bibliographic research with a qualitative approach, with the objective of describing the coping of cancer patients in the face of chemotherapy: nursing contributions. After the association of all descriptors, 151,280 articles were found, 151,266 were excluded and selected 14. In view of the nursing contributions, it is necessary to identify the fundamental particularities of each patient, for a comprehensive contribution and efficient therapeutic results. After reflective reading, two categories emerged: Nursing contributions to coping with oncology patients in the face of chemotherapy and Optics of the oncology patients in the face of chemotherapy. Nursing has a fundamental role in the face of the paradigms that permeate the confrontation of the individual affected by the oncological disease, since in most cases the patient primarily needs assistance support. It is concluded that it is extremely important to evaluate the main factors that can contribute to the suffering of cancer patients in the face of chemotherapy-based treatment, for better professional performance and quality of care provided to the patient.
\end{abstract}

Keywords: Nursing; Chemotherapy; Oncology.

\title{
Resumen
}

El cáncer ocurre cuando el aumento de células corporales está fuera de control y en consecuencia la quimioterapia es uno de los tratamientos más utilizados para el dominio sistémico de la enfermedad, ya que esta alternativa está siendo muy relevante en el combate de la enfermedad de forma singular o combinada sin embargo, es capaz de provocar de gran manera la debilidad física y emocional del individuo. Se trata de una investigación bibliográfica con abordaje cualitativo, con el objetivo de describir el afrontamiento de los pacientes oncológicos. Luego de la asociación de todos los descriptores, se encontraron 151.280 artículos, se excluyeron y seleccionaron 151.266. En vista de los aportes de enfermería, es necesario identificar las particularidades fundamentales de cada paciente, para una contribución integral y resultados terapéuticos eficientes. Luego de la lectura reflexiva surgieron dos categorías: Aportes de enfermería al afrontamiento del paciente oncológico ante la quimioterapia y Óptica del paciente oncológico ante la quimioterapia. La enfermería tiene un papel fundamental frente a los paradigmas que impregnan el enfrentamiento del individuo afectado por la enfermedad oncológica, ya que en la mayoría de los casos el paciente necesita principalmente el apoyo asistencial. Concluimos que es de suma importancia evaluar los principales factores que pueden contribuir al sufrimiento del paciente oncológico frente al tratamiento quimioterápico, para un mejor desempeño profesional y calidad de la atención brindada al paciente.

Palabras clave: Enfermería; Quimioterapia; Oncología.

\section{Introdução}

O câncer é uma patologia que tem como característica o crescimento anormal de células, destruindo o tecido do corpo, podendo invadir regiões distantes da origem do tumor (metástase). Sendo assim, essa doença é responsável por causar fragilidade emocional e física no indivíduo. O tumor maligno é a segunda principal causa de morte no mundo, constituindo um problema de saúde pública universal, sendo responsável por 9,6 milhões de mortes em 2018 (Nascimento et al.,2020).

Acerca de uma doença oncológica, a sua adesão pode estar relacionada a fatores hereditários ou até mesmo ambientais, que contribuem para o desenvolvimento da doença, como: má alimentação, sedentarismo, uso de álcool e drogas. Condições essas, que refletem no bem-estar do paciente e precisam ser analisadas com êxito pelo enfermeiro que tem um papel fundamental diante dessas circunstâncias (Silva et al., 2019).

A descoberta de um câncer, seguido da necessidade de realizar um tratamento, causam emoções e reações no paciente, como também a necessidade de modificações na rotina diária da vida. Para que toda essa mudança ocorra, o enfermeiro tem importante papel neste momento, já que é de extrema relevância que ele realize intervenções junto com a equipe multidisciplinar e familiares do paciente, com caráter de acolhimento e visando a melhoria da qualidade de vida, uma vez que, a desordem emocional e nas características físicas devido a quimioterapia, podem causar um transtorno no processo de enfrentamento da doença (Oliveira et al., 2018). 
Nesse sentido, a quimioterapia é utilizada para controle sistêmico do câncer, sendo cada vez mais relevante no combate à doença de forma singularizada ou combinada. Com isso, os quimioterápicos interferem na capacidade de multiplicação de células cancerosas com finalidades curativas ou paliativas (Silva et al., 2019).

$\mathrm{O}$ tratamento à base de quimioterápicos afeta psicologicamente o paciente e sua família, causando uma mudança de comportamento e alterações na sua autoestima. Nesse caso, é necessário o apoio familiar e da equipe multidisciplinar para o enfrentamento da enfermidade e uma melhor qualidade de vida, de modo que, o doente não venha a desistir do tratamento (Leite et al., 2015).

É necessário identificar todas as barreiras que podem influenciar e dificultar o tratamento do paciente, pois é imprescindível proporcionar intervenções terapêuticas e apoio adequado em tempo oportuno, garantindo atendimento contínuo e eficaz. Nessa situação, é vultoso saber como uma pessoa com diagnóstico de tumor maligno reage ao tratamento identificando os sintomas e dificuldades vivenciadas pelo doente (Teston et al., 2018).

Segundo Silva e Moreira (2018), é de extrema importância saber observar o grau de complexidade de um paciente oncológico, para uma abordagem e continuidade das intervenções terapêuticas de acordo com suas reais necessidades, portanto, a assistência prestada ao paciente deve seguir uma programação adequada e segura.

Diante dos recursos utilizados no tratamento de neoplasias, a quimioterapia tem a maior incidência de cura, utilizando compostos químicos no intuito de aumentar a sobrevida de portadores dessa patologia. Por conseguinte, no período de tratamento é necessário identificar todas as dificuldades do enfermo, afim de proporcionar uma assistência afetiva (Souza et al., 2016).

De todos os profissionais da equipe multidisciplinar na saúde, a enfermagem tem função primordial para lidar com o enfrentamento de uma pessoa portadora de câncer, utilizando estratégias que visam amenizar desconforto emocional e físico, afim de promover qualidade de vida (Macedo et al., 2019).

Partindo desse princípio, a oncologia se torna uma particularidade que requer alta demanda da assistência da enfermagem, atuando de forma empática e afetiva. Com isso, os profissionais dessa área, tem contato direto com a dor e reações inesperadas no processo de desesperança do paciente (Lins \& Souza, 2018).

Imagina-se que o cuidar em enfermagem está agregado às lamúrias do paciente que necessitam ser identificadas pela equipe precocemente. Neste sentido, o enfermeiro atinge importante grau de proximidade do doente, que lhe confere a capacidade de destacar as principais contrariedades no processo terapêutico (Stube et al., 2015).

Assim sendo, para uma melhor contribuição no combate ao câncer pelos profissionais de enfermagem, estes, necessitam estar preparados emocionalmente para amparar o paciente em qualquer circunstância, criando então, um vínculo afetivo e extremamente profissional (Bubolz et al., 2019).

Por estas razões, a equipe de enfermagem precisa estar corretamente disposta para realizar todos os procedimentos indispensáveis, referente a administração de quimioterápicos e avaliação integral do paciente e seus familiares, para melhor lidar com as complicações do tratamento oncológico (Rodrigues et al., 2019).

Diante da imprecisão no ramo de cuidados terapêuticos e impacto da evolução das células cancerígenas no organismo, o paciente se torna vulnerável à sentimentos negativos, refletindo então no processo de tomada de decisão, relativo à obtenção dos recursos disponíveis no combate ao câncer (Carmo et al.,2019).

Segundo o Datasus (2016), em 2015 foram realizados no Brasil 5,7 milhões de procedimentos oncológicos pelo Sistema Único de Saúde (SUS). Por conseguinte, ocorreu um crescimento de 38\% em cinco anos, de quimioterapias feitas pelo SUS, sendo 2.190.734 em 2010, evoluindo para 2.903.139 em 2015. 
Frente a isso emergiu as seguintes questões norteadoras: Quais as contribuições da enfermagem no enfrentamento do paciente oncológico diante da quimioterapia e como o paciente oncológico vislumbra o processo de enfrentamento da quimioterapia?

O presente estudo tem como objetivo descrever as contribuições da enfermagem no enfrentamento do paciente oncológico diante da quimioterapia e identificar através da literatura, como o paciente oncológico vislumbra o tratamento contra o câncer. Dessa forma, se torna possível que o enfermeiro, junto a equipe multidisciplinar de saúde minimizem o impacto causado pela quimioterapia e/ou outro tipo de tratamento que pode estar associado (Wakiuchi et al., 2018).

\section{Metodologia}

Segundo Lakatos e Marcone (2003), conhecimento científico determina a utilização de métodos científicos; por outro lado, não são todos os estudos que utilizam esse modelo é reconhecido como ciência.

Perante a certificação, pode-se deduzir que a aplicação de métodos científicos não é competência especifica da ciência, com tudo não existe ciência sem o uso de métodos científicos. Como tal característica, o método é a agregação de atividades sistemáticas e lógicas que, permite com total segurança e economia, atingir o objetivo, com estudos validos e verdadeiros, elaborando roteiros a serem seguidos, encontrando erros e contribuindo com soluções dos cientistas (Lakatos \& Marcone, 2003).

$\mathrm{Na}$ atualidade têm-se uma farta e complexa quantidade de dados na área da saúde, fazendo assim, com que haja necessidade de desenvolvimento de artigos e pesquisas, com embasamento cientifico, para possibilitar melhor delimitação metodológica esclarecendo diversos estudos. Mediante a necessidade, utilizamos a revisão bibliográfica como uma forma de metodologia que possibilita um apanhado de conhecimentos e aplica-se em resultados de estudos concisos na pratica do profissional (Minayo, 2013).

Trata-se de um estudo de revisão bibliográfica, de caráter descritivo e de abordagem qualitativa. A pesquisa científica é a atuação básica das ciências na sua indagação e construção da realidade, tornando-a uma atividade expressiva (Minayo, 2013).

Abordagem qualitativa é aquela que não trabalha com informações numéricas, mas sim, que trabalha com conceitos, ideologias, processos de comunicação humana, entre outros. E apresenta facilidade de definir hipótese ou problema, de explorar a interação de certas variáveis, de compreender e classificar processos dinâmicos experimentados por grupos sociais, de apresentar mudanças, elaboração ou formação de posição de determinados grupos, e de permitir, em grau de profundidade, a interpretação dos comportamentos ou atitudes dos indivíduos (Gil, 2008).

Foram realizadas buscas na base de dados da Biblioteca Virtual em Saúde (BVS) - Bireme, entre junho e novembro, 2019, nas bases de dados: Literatura Latico Americana e do Caribe em Ciência da Saúde (LILACS), Bases de Dados da Enfermagem (BDENF), Biblioteca Eletrônica Cientifica Online (SciELO).

Para a busca das referências foram utilizados os descritores "enfermagem", "quimioterapia", "oncologia", advindos do sistema de Descritores em ciências da saúde (DeCS), utilizando o marcador "AND”. Para resgaste dos artigos, consideramos como critérios para inclusão artigos publicados no período compreendido entre os anos 2015 e 2019 com textos completos em língua portuguesa. E os critérios de exclusão foram os artigos repetidos, publicações com textos não disponíveis, fora da língua vernácula e estudos com mais de cinco anos de publicação.

Inicialmente foram pesquisados os descritores individualmente, sendo encontrados artigos científicos conforme Quadro 1, a seguir: 
Quadro 1: Descritores Isolados.

\begin{tabular}{|c|c|c|c|c|c|c|}
\hline Descritores & BDENF & LILACS & MEDLINE & $\begin{array}{c}\text { Google } \\
\text { Acadêmico }\end{array}$ & $\begin{array}{c}\text { Artigos } \\
\text { Encontrados }\end{array}$ & $\begin{array}{c}\text { Artigos } \\
\text { Selecionados }\end{array}$ \\
\hline Enfermagem & 5.951 & 5.889 & 1.291 & 61.400 & 74.531 & 1 \\
\hline Quimioterapia & 134 & 721 & 709 & 14.900 & 16.464 & 0 \\
\hline Oncologia & 170 & 422 & 50 & 15.700 & 16.464 & 4 \\
\hline
\end{tabular}

Fonte: Autores.

Diante do extenso número de publicações encontradas, realizou-se um refinamento na busca. Os descritores foram pesquisados de forma associada em dupla, utilizando o termo "and", conforme Quadro 2:

Quadro 2: Distribuição quantitativa das produções científicas encontradas nas bases de dados com descritores associados em dupla.

\begin{tabular}{|c|c|c|c|c|c|c|}
\hline \multicolumn{7}{|c|}{ Banco de Dados } \\
\hline Descritores & BDENF & LILACS & MEDLINE & $\begin{array}{c}\text { Google } \\
\text { Acadêmico }\end{array}$ & $\begin{array}{c}\text { Artigos } \\
\text { Encontrados }\end{array}$ & $\begin{array}{c}\text { Artigos } \\
\text { Selecionados }\end{array}$ \\
\hline $\begin{array}{c}\text { Enfermagem and } \\
\text { quimioterapia }\end{array}$ & 102 & 111 & 23 & 9.930 & 10.166 & 0 \\
\hline $\begin{array}{c}\text { Enfermagem and } \\
\text { oncologia }\end{array}$ & 136 & 130 & 12 & 15.200 & 15.478 & 6 \\
\hline $\begin{array}{c}\text { Quimioterapia and } \\
\text { oncologia }\end{array}$ & 17 & 76 & 12 & 11.90 & 12.005 & 1 \\
\hline
\end{tabular}

Fonte: Autores.

Considerando ainda ser extensa a quantidade de produções científicas, optou-se pela busca com os descritores associados em trio. Os resultados dessa busca se encontram descritos no Quadro 3.

Quadro 3: Distribuição quantitativa das produções científicas encontradas nas bases de dados com os descritores associados em trio.

\begin{tabular}{|c|c|c|c|c|c|c|}
\hline \multicolumn{2}{|c|}{ Banco De Dados } \\
\hline Descritores & BDENF & LILACS & MEDLINE & $\begin{array}{c}\text { Google } \\
\text { Acadêmic } \\
\text { o }\end{array}$ & $\begin{array}{c}\text { Artigos } \\
\text { Encontrados }\end{array}$ & $\begin{array}{c}\text { Artigos } \\
\text { Selecionados }\end{array}$ \\
\hline $\begin{array}{c}\text { Enfermagem and } \\
\text { quimioterapia and } \\
\text { oncologia }\end{array}$ & 14 & 18 & 2 & 6.260 & 6.294 & 3 \\
\hline
\end{tabular}

Fonte: Autores.

Finalizado esse percurso de busca, realizou-se a leitura dos resumos e os que apresentavam relevância para subsidiar a discussão do tema foram selecionados e lidos na íntegra. 
A partir dessa leitura preliminar, foram selecionados 14 artigos que mantinham coerência com os descritores acima apresentados e com os objetivos do estudo. A partir dessa análise, foi extraída a bibliografia potencial, explicitada no Quadro 4 a seguir:

Quadro 4: Levantamento estrutural dos artigos selecionados nas bases de dados da temática.

\begin{tabular}{|c|c|c|c|c|c|}
\hline Título & Autores & Objetivo & Revista & Ano & Principais conclusões \\
\hline $\begin{array}{c}\text { Avaliação da } \\
\text { autoestima em pacientes } \\
\text { oncológicos submetidos } \\
\text { a tratamento } \\
\text { quimioterápico. }\end{array}$ & Leite et al. & $\begin{array}{l}\text { Avaliar a autoestima de } \\
\text { pacientes oncológicos } \\
\text { submetidos a } \\
\text { quimioterapia. }\end{array}$ & $\begin{array}{l}\text { Revista Latino- } \\
\text { Am.Enfermagem }\end{array}$ & 2015 & $\begin{array}{l}\text { Os pacientes oncológicos } \\
\text { avaliados apresentaram } \\
\text { autoestima alta; assim, } \\
\text { torna-se fundamental que a } \\
\text { enfermagem planeje a } \\
\text { assistência dos pacientes } \\
\text { em tratamento } \\
\text { quimioterápico }\end{array}$ \\
\hline $\begin{array}{c}\text { Percepções de } \\
\text { enfermeiros e manejo } \\
\text { da dor de pacientes } \\
\text { oncológicos. }\end{array}$ & Stube et al. & $\begin{array}{l}\text { Apreender a percepção } \\
\text { de enfermeiros que } \\
\text { atuam em Oncologia } \\
\text { referente à dor do } \\
\text { paciente e conhecer } \\
\text { ações para seu manejo. }\end{array}$ & $\begin{array}{l}\text { Revista Mineira de } \\
\text { Enfermagem }\end{array}$ & 2015 & $\begin{array}{c}\text { Podem ser realizadas } \\
\text { mudanças por enfermeiros, } \\
\text { com o objetivo de qualificar } \\
\text { a assistência aos pacientes } \\
\text { oncológicos e contribuir } \\
\text { para a minimização da dor. }\end{array}$ \\
\hline $\begin{array}{l}\text { Emergência oncológica: } \\
\text { atuação dos enfermeiros } \\
\text { no extravasamento de } \\
\text { drogas quimioterápicas } \\
\text { antineoplásicas }\end{array}$ & Souza et al. & $\begin{array}{l}\text { Investigar a atuação dos } \\
\text { enfermeiros no } \\
\text { extravasamento de } \\
\text { quimioterápicos } \\
\text { antineoplásicos. }\end{array}$ & $\begin{array}{l}\text { Esc. Anna Nery } \\
\text { Rev. Enfermagem }\end{array}$ & 2016 & $\begin{array}{l}\text { Os resultados encontrados } \\
\text { são importantes, vez que } \\
\text { apontam para questões que } \\
\text { devem ser refletidas por } \\
\text { gestores hospitalares e de } \\
\text { instituições formadoras. }\end{array}$ \\
\hline $\begin{array}{l}\text { Oncologia: avanços e } \\
\text { desafios para o SUS }\end{array}$ & Barros & $\begin{array}{l}\text { Enfatizar os avanços e } \\
\text { desafios para o SUS. }\end{array}$ & DATASUS & 2016 & $\begin{array}{c}\text { Dar preferência às } \\
\text { habilitações de novos } \\
\text { serviços, para garantir um } \\
\text { atendimento mais próximo } \\
\text { da população. }\end{array}$ \\
\hline $\begin{array}{c}\text { Sentimentos e } \\
\text { dificuldades } \\
\text { vivenciadas por } \\
\text { pacientes oncológicos } \\
\text { ao longo dos itinerários } \\
\text { diagnóstico e } \\
\text { terapêuticos. }\end{array}$ & Teston et al. & $\begin{array}{l}\text { Compreender os } \\
\text { sentimentos e } \\
\text { dificuldades } \\
\text { vivenciadas por } \\
\text { pacientes oncológicos } \\
\text { frente aos itinerários } \\
\text { diagnostico e } \\
\text { terapêutico. }\end{array}$ & $\begin{array}{l}\text { Esc. Anna Nery } \\
\text { Rev. Enfermagem }\end{array}$ & 2018 & $\begin{array}{l}\text { Compreendeu-se que } \\
\text { durante a vivencia do } \\
\text { itinerário diagnóstico e } \\
\text { terapêutico as pessoas com } \\
\text { câncer experimentam } \\
\text { sentimentos negativos e } \\
\text { muitas dificuldades. }\end{array}$ \\
\hline $\begin{array}{l}\text { Grau de complexidade } \\
\text { dos cuidados de } \\
\text { enfermagem: } \\
\text { readmissões } \\
\text { hospitalares de pessoas } \\
\text { com câncer de mama }\end{array}$ & $\begin{array}{c}\text { Silva e } \\
\text { Moreira }\end{array}$ & $\begin{array}{c}\text { Avaliar o grau de } \\
\text { complexidade dos } \\
\text { cuidados de } \\
\text { enfermagem de } \\
\text { pacientes com câncer de } \\
\text { mama readmitidas na } \\
\text { oncologia clínica. } \\
\end{array}$ & $\begin{array}{l}\text { Revista Gaúcha de } \\
\text { Enfermagem }\end{array}$ & 2018 & $\begin{array}{l}\text { O grau de complexidade } \\
\text { dos pacientes readmitidos } \\
\text { foi predominantemente } \\
\text { semi-intensivo e intensivo. }\end{array}$ \\
\hline $\begin{array}{c}\text { Formação dos } \\
\text { enfermeiros para o } \\
\text { cuidado em oncologia }\end{array}$ & Lins e Souza & $\begin{array}{l}\text { Analisar os aspectos } \\
\text { relacionados à formação } \\
\text { dos enfermeiros } \\
\text { residentes, às } \\
\text { dificuldades e } \\
\text { facilidades para o } \\
\text { cuidado em oncologia. }\end{array}$ & $\begin{array}{c}\text { Revista de } \\
\text { Enfermagem UFPE }\end{array}$ & 2018 & $\begin{array}{c}\text { Foi possível identificar que } \\
\text { a formação dos enfermeiros } \\
\text { para o cuidado em } \\
\text { oncologia ainda é } \\
\text { insipiente. Tal fato foi } \\
\text { evidenciado pelas } \\
\text { dificuldades como a falta de } \\
\text { embasamento teórico e o } \\
\text { curto período de estágio. }\end{array}$ \\
\hline
\end{tabular}




\begin{tabular}{|c|c|c|c|c|c|}
\hline $\begin{array}{c}\text { Busca por cuidado } \\
\text { oncológico: percepção } \\
\text { de pacientes e } \\
\text { familiares }\end{array}$ & Oliveira et al. & $\begin{array}{l}\text { Compreender a busca } \\
\text { por cuidado do câncer a } \\
\text { partir da percepção de } \\
\text { pacientes e familiares/ } \\
\text { cuidadores. }\end{array}$ & $\begin{array}{c}\text { Revista de } \\
\text { Enfermagem UFPE }\end{array}$ & 2018 & $\begin{array}{c}\text { Pacientes e familiares } \\
\text { enfrentam diversas } \\
\text { dificuldades para realizar a } \\
\text { busca por cuidado do } \\
\text { câncer desde a etapa inicial, } \\
\text { para a obtenção do } \\
\text { diagnóstico de câncer, até o } \\
\text { final do tratamento. }\end{array}$ \\
\hline $\begin{array}{l}\text { Sentidos e dimensões } \\
\text { do câncer por pessoas } \\
\text { adoecidas: } \\
\text { análise estrutural das } \\
\text { representações sociais }\end{array}$ & $\begin{array}{c}\text { Wakiuchi et } \\
\text { al. }\end{array}$ & $\begin{array}{l}\text { Descrever os conteúdos } \\
\text { e a estrutura da } \\
\text { representação social do } \\
\text { câncer. }\end{array}$ & $\begin{array}{l}\text { Revista da Escola } \\
\text { de Enfermagem da } \\
\text { USP }\end{array}$ & 2018 & $\begin{array}{l}\text { As representações sociais } \\
\text { do câncer, a partir das inter- } \\
\text { relações apresentadas, } \\
\text { propiciam reflexões que } \\
\text { podem contribuir para o } \\
\text { incremento do cuidado } \\
\text { individual e social do } \\
\text { paciente com neoplasia } \\
\text { maligna e sua família, nos } \\
\text { serviços de saúde. }\end{array}$ \\
\hline $\begin{array}{l}\text { Abordagem educativa } \\
\text { ao paciente oncológico: } \\
\text { estratégias para } \\
\text { orientação acerca do } \\
\text { tratamento } \\
\text { quimioterápico. }\end{array}$ & Silva et al. & $\begin{array}{c}\text { Propor um modelo de } \\
\text { orientação ao paciente } \\
\text { oncológico acerca do } \\
\text { tratamento } \\
\text { quimioterápico, por } \\
\text { meio de um informativo } \\
\text { impresso e da criação } \\
\text { do diário do paciente. }\end{array}$ & $\begin{array}{l}\text { Revista brasileira de } \\
\text { cancerologia }\end{array}$ & 2019 & $\begin{array}{l}\text { A proposta e a elaboração } \\
\text { dos instrumentos foram } \\
\text { estratégias de grande } \\
\text { relevância na educação em } \\
\text { saúde ao paciente, uma vez } \\
\text { que auxiliam e } \\
\text { instrumentalizam o } \\
\text { profissional nesse processo } \\
\text { e possibilitam que o } \\
\text { paciente participe } \\
\text { ativamente de seu } \\
\text { tratamento. }\end{array}$ \\
\hline $\begin{array}{c}\text { Estratégias de } \\
\text { enfrentamento dos } \\
\text { profissionais de } \\
\text { enfermagem frente à } \\
\text { morte na oncologia } \\
\text { pediátrica: revisão } \\
\text { integrativa }\end{array}$ & Macedo et al. & $\begin{array}{c}\text { Identificar as estratégias } \\
\text { de enfrentamento } \\
\text { utilizadas por } \\
\text { profissionais de } \\
\text { enfermagem que atuam } \\
\text { na oncologia pediátrica } \\
\text { diante da morte do } \\
\text { paciente. }\end{array}$ & $\begin{array}{c}\text { Revista Online de } \\
\text { Pesquisa: } \\
\text { Cuidado é } \\
\text { Fundamental }\end{array}$ & 2019 & $\begin{array}{c}\text { As estratégias levam a } \\
\text { atitudes auxiliadoras nas } \\
\text { necessidades do } \\
\text { profissional, amenizando } \\
\text { seu sofrimento e } \\
\text { melhorando seu processo de } \\
\text { cuidar. }\end{array}$ \\
\hline $\begin{array}{c}\text { Percepções dos } \\
\text { profissionais da } \\
\text { enfermagem a respeito } \\
\text { do sofrimento e das } \\
\text { estratégias de } \\
\text { enfrentamento na } \\
\text { Oncologia }\end{array}$ & Bubolz et al. & $\begin{array}{c}\text { Conhecer quais as } \\
\text { situações que causam } \\
\text { prazer e sofrimento no } \\
\text { trabalho dos } \\
\text { profissionais de } \\
\text { enfermagem em setores } \\
\text { oncológicos. }\end{array}$ & $\begin{array}{l}\text { Revista online de } \\
\text { pesquisa: Cuidado é } \\
\text { Fundamental }\end{array}$ & 2019 & $\begin{array}{c}\text { O profissional de } \\
\text { enfermagem está rodeado } \\
\text { tanto de sentimento de } \\
\text { prazer no setor oncológico, } \\
\text { quanto de sofrimento } \\
\text { relacionado } \\
\text { preponderantemente pela } \\
\text { morte do paciente. }\end{array}$ \\
\hline $\begin{array}{c}\text { Cuidar em oncologia: } \\
\text { desafios e superações } \\
\text { cotidianas vivenciados } \\
\text { por enfermeiros }\end{array}$ & Carmo et al. & $\begin{array}{c}\text { Compreender a } \\
\text { perspectiva de } \\
\text { enfermeiros acerca do } \\
\text { processo de } \\
\text { enfrentamento dos } \\
\text { desafios vivenciados no } \\
\text { cuidado à pessoa com } \\
\text { câncer. }\end{array}$ & $\begin{array}{l}\text { Revista brasileira de } \\
\text { cancerologia }\end{array}$ & 2019 & $\begin{array}{l}\text { Os resultados apontam que } \\
\text { é necessário reconduzir um } \\
\text { novo olhar à formação de } \\
\text { profissionais de saúde, para } \\
\text { além das competências } \\
\text { técnicas. }\end{array}$ \\
\hline $\begin{array}{l}\text { Atitudes de enfermeiros } \\
\text { na administração de } \\
\text { quimioterápicos em } \\
\text { oncologia pediátrica }\end{array}$ & $\begin{array}{l}\text { Rodrigues et } \\
\text { al. }\end{array}$ & $\begin{array}{l}\text { Analisar as atitudes de } \\
\text { enfermeiros acerca da } \\
\text { administração de } \\
\text { antineoplásicos em } \\
\text { oncologia pediátrica. }\end{array}$ & $\begin{array}{c}\text { Revista } \\
\text { Enfermagem UERJ }\end{array}$ & 2019 & $\begin{array}{c}\text { Todos os enfermeiros } \\
\text { consideraram a QT o } \\
\text { principal tratamento para o } \\
\text { câncer infanto-juvenil. }\end{array}$ \\
\hline
\end{tabular}




\begin{tabular}{|c|c|c|c|c|c|}
\hline $\begin{array}{c}\text { Câncer infantojuvenil: } \\
\text { Perfil dos pacientes } \\
\text { atendidos na unidade de } \\
\text { alta complexidade em } \\
\text { oncologia (UNACON) } \\
\text { em Rio Branco-Acre }\end{array}$ & $\begin{array}{c}\text { Nascimento et } \\
\text { al. }\end{array}$ & $\begin{array}{c}\text { Descrever o perfil } \\
\text { clínico } \\
\text { e epidemiológico de } \\
\text { crianças e adolescentes } \\
\text { atendidos na UNACON } \\
\text { durante o ano de 2017. }\end{array}$ & $\begin{array}{c}\text { Arquivos de } \\
\text { Ciências da Saúde } \\
\text { da UNIPAR }\end{array}$ & $\begin{array}{c}\text { Conhecer o perfil pode } \\
\text { contribuir para a tomada de } \\
\text { decisóes da equipe e no } \\
\text { estabelecimento de medidas } \\
\text { assistenciais aos pacientes, } \\
\text { visando um atendimento } \\
\text { humanizado, voltado para } \\
\text { as necessidades } \\
\text { sociodemográficas e as } \\
\text { características clínicas- } \\
\text { epidemiológicas do grupo } \\
\text { populacional. }\end{array}$ \\
\hline
\end{tabular}

Fonte: Autores.

Foram elaboradas às categorias de análise e sucedemos para a fase final de inferência e discussão dos dados obtidos, mediante o respaldo alcançado através da articulação entre o conteúdo verificado nas produções científicas e a atitude críticoreflexiva das pesquisadoras, através da qual identificamos a protagonização do enfermeiro na assistência ao paciente oncológico paliativo.

\section{Resultados e Discussão}

Posterior à leitura reflexiva emergiram duas categorias: Contribuições da enfermagem no enfrentamento do paciente oncológico diante da quimioterapia e ótica do paciente oncológico frente a quimioterapia.

\section{Categoria 1 - Contribuições da enfermagem no enfrentamento do paciente oncológico diante da quimioterapia.}

Os enfermeiros participam do tratamento de pacientes oncológicos de forma integral, podendo assim, comprometer sua estrutura emocional, uma vez que, as medidas de intervenção relacionadas ao câncer se tornam sacrificantes ao doente devido as dificuldades e mudanças, tornando necessário que a equipe de enfermagem tenha um amplo preparo emocional para lidar com as emoções do cliente e principalmente de sua família que também necessita de um apoio psicológico eficaz para lidar com as novas mudanças no cotidiano ao lado do paciente e ter segurança referente às reações adversas que podem ocorrer (Carmo et al., 2019).

O câncer está diretamente relacionado à dor, que inclusive deve ser evidenciada pelo enfermeiro, para que seja possível direcionar estratégias e intervenções para o seu controle e realizar inspeção de resultados para níveis considerados toleráveis pelo paciente, uma vez que a dor pode interferir no âmbito fisiológico, psíquico, social e espiritual do paciente, pois se trata de uma experiência sensitiva e emocional desagradável associada a uma lesão real ou potencial dos tecidos (Stube $e t$ al., 2015).

Diante disso, a enfermagem pode se deparar com diversas circunstâncias relacionadas a nova rotina de vida, que pode desencadear desgaste físico e emocional, estando assim mais vulnerável ao sofrimento no trabalho. Nesta perspectiva, é de suma importância que os profissionais estejam realizados e satisfeitos com a prática exercida garantindo a confiança e satisfação do paciente e também de seus familiares que devem receber orientações eficientes, pois estes acompanham toda a trajetória do doente, desde os procedimentos diagnósticos até a fase final da doença (Bulboz et al., 2019).

Sendo assim, a enfermagem constrói um vínculo com o adoentado, permitindo o desenvolvimento de uma relação que vai além do cuidado direto em todas as fases da doença que exige recursos materiais e terapêuticos específicos, portanto, o cuidador em questão pode se encarregar de uma rotina mais desgastante, assim trazendo para si um possível abalo emocional (Macedo et al., 2019). 
É essencial que a elaboração do cuidado prestado pela equipe de enfermagem seja atrelado às principais necessidades do paciente oncológico, assegurando recursos para uma assistência de qualidade e segura, representando assim um segmento de extrema relevância no âmbito hospitalar, visto que a enfermagem tem um importante papel no gerenciamento dos cuidados prestados (Silva \& Moreira, 2018).

Com isso, se torna imprescindível destacar a importância do conhecimento concernente às intervenções para uma atuação assistencial e gerencial efetiva da enfermagem, visto que, o enfermeiro requer domínio e habilidades específicas no que se refere ao manuseio e administração de antineoplásicos, consequentemente, acarretando uma detecção precoce de complicações, evitando prejuízos ao paciente (Souza et al., 2016).

De todos os cuidados essenciais, a administração de quimioterápicos é indispensável e requer a inclusão do enfermeiro para que todo processo seja seguro, concedendo um amparo necessário e habilitando o paciente e seus familiares para lidar com todas as atribulações que possam surgir no processo terapêutico como um todo e capacitando o enfermo e sua família para lidar com a nova rotina de vida nesta modalidade de tratamento (Rodrigues et al., 2019).

$\mathrm{Na}$ oncologia, a enfermagem além de realizar assistência durante todo o processo terapêutico do paciente, precisa-se que toda a equipe de saúde tenha capacidade relacional e afetiva, devido as especificidades do cliente, como situações de dor; efeitos colaterais; reações físicas e emocionais; expectativas de curas, causando a necessidade do apoio da equipe de enfermagem no enfrentamento do paciente (Lins \& Souza, 2018).

\section{Categoria 2 - Ótica do paciente oncológico frente a quimioterapia}

Diante do exposto, se torna necessário observar a percepção que o indivíduo acometido pelo câncer tem sobre a sua real situação, nova rotina e hábitos de vida, pois além de um diagnóstico precoce e tratamento condizente, é preciso ter um bom índice de qualidade de vida e emocional saudável. Nessas condições se torna possível melhor satisfação no processo terapêutico e confiança no tratamento realizado (Leite et al., 2015).

Frente a esse cenário, se torna indispensável conhecer como a pessoa com câncer padece diante dos itinerários diagnóstico e terapêutico. Pois assim, é possível realizar uma assistência de saúde condizente com a realidade de um paciente oncológico. Por consequência, compreendendo cada sentimento e dificuldade demonstrados e observando todas as atitudes vivenciadas em todo o processo de tratamento (Teston et al., 2018).

Destaca-se que todo o fortalecimento na sobrevida da pessoa com câncer é devido o avanço tecnológico dos meios diagnósticos e terapêuticos. Destarte, todas as ações relacionadas às intervenções para a melhoria da qualidade de vida do doente é devido à preocupação dos profissionais com a reabilitação e tratamento do indivíduo que se encontra debilitado (Teston et al., 2018).

Em todo o âmbito terapêutico do indivíduo com câncer é possível que toda a equipe de saúde envolvida vivencie o sofrimento, angústia, insegurança, dor e até mesmo revolta devido às incertezas relacionadas à doença, no que diz respeito a sua evolução e os resultados do tratamento. Essa situação referente aos sentimentos e comportamento do paciente é devido a sua ótica de que o câncer não tem cura (Carmo et al., 2019).

A autoimagem do paciente é um dos fatores mais acometidos pela doença oncológica, devido às limitações físicas que ocorrem no decorrer do tratamento para a melhoria da qualidade de vida. Com isso, se torna necessário que o paciente tenha um processo de adaptação ou ajuste psicossocial, para lidar com a nova situação que se encontra e impasses específicos referente a doença, tendo assim, capacidade para lidar com seu sofrimento e melhora na autoestima (Leite et al., 2015).

Em algumas situações a pronúncia câncer é reprimida por alguns pacientes oncológicos, na tentativa de negar a patologia ou tentar se afastar do sofrimento que ela traz e com isso acreditam que essa doença se trata de um castigo, punição 
ou algo devastador para as suas vidas e evidenciam dimensões preponderantemente negativas, e ter pensamentos sobre adoecimento, a vida e a morte, tornam-se frequente (Wakiuchi et al., 2018).

Ademais, por mais que o câncer não esteja necessariamente associado à morte, para os pacientes ainda representa uma ameaça, afetando o bem-estar de pessoas debilitadas. O medo do câncer está presente em maior grau entre pessoas que já foram diagnosticadas com a doença. Relacionado ao que é difícil, a doença oncológica associa-se à dificuldade de controle da patologia e sobrevida a partir do diagnóstico (Wakiuchi et al., 2018).

No processo de busca pelo cuidado oncológico ocorrem diversos percalços que comprometem o comportamento e aderência do paciente ao tratamento e apoio da família em todo o processo do cuidado, desde do início da terapia e durante o decorrer, como: isolamento social, deixar de realizar momentos de lazer, transformações negativas nas relações e comprometimento de aspectos físicos e emocionais (Oliveira et al., 2018).

\section{Considerações Finais}

Conclui-se, diante do exposto, que o ramo da oncologia vai muito além do cuidado direto da equipe de saúde com o paciente, e de fato a enfermagem ganha destaque perante todo o tratamento, devido à realização de ações integradas com outros profissionais para reabilitação do doente, intervenções nas reações do tratamento e atuação como educador dos familiares, que também necessitam de um acompanhamento rigoroso, perante todas as adversidades.

É preciso identificar os fatores que contribuem ou agravam o sofrimento do indivíduo que adquiriu uma neoplasia, tornando ainda mais coerente o processo de compreensão e complacência da enfermagem, pois diversas condições podem estar englobadas no processo de adesão do cliente diante da quimioterapia, sendo esta, umas das terapias mais utilizadas como método curativo da doença, todavia devido aos efeitos adversos, se torna desgastante para o paciente.

Uma vez identificadas as causas do sofrimento, a enfermagem pode intervir com métodos pertinentes e mais eficazes perante as contrariedades que dificultam o desempenho de uma assistência de qualidade, enfatizando os impasses mais relevantes. Impasses esses, que podem estar ligados à autoimagem, dificuldade de adaptação com o diagnóstico, mudança nos hábitos alimentares e comportamentais, afastamento social e modificação na rotina do cotidiano.

Espera-se contribuir com este estudo, para o incentivo da enfermagem em investir no aperfeiçoamento da busca pela identificação de razões que possam favorecer a negação do paciente diante do âmbito terapêutico, bem como estorvar o processo de cura. Com o desenvolvimento dos objetivos propostos, torna-se favorável o aprimoramento das técnicas profissionais realizadas e por conseguinte aquisição da confiança e afeição do paciente e familiares.

Portanto, a equipe de enfermagem necessita acompanhar o paciente oncológico de maneira integral, sempre com ênfase em uma comunicação efetiva sobre a terapia e sua toxicidade, com o doente e sua família que acompanhou todo o processo terapêutico. Com isso, independente da fase de tratamento em que se encontra o indivíduo com câncer, ele e seus familiares terão instrumentação para enfrentar possíveis ocorrências de agravos.

\section{Referências}

Bubolz, B. K. et al. (2019). Percepções dos Profissionais da Enfermagem a Respeito do Sofrimento e das Estratégias de Enfrentamento na Oncologia. Rev. pesqui. cuid. fundam. (Online), 599-606.

Carmo, R. A. L. O., Siman, G. A., Matos, R. A. \& Mendonça, E. T. (2019). Cuidar em Oncologia: Desafios e Superações Cotidianas Vivenciados por Enfermeiros. Revista Brasileira de Cancerologia, 65(3).

Datasus. Oncologia: avanços e desafios para o SUS. file://C:/Users/Meu/Documents/a\%20TCC/TCC\%20ONCO/ARTIGOS\%20PARA\%20O\%20 TCC\%20ONCO/artigos\%20gogle\%20academico\%20utilizados/Forum-Estadao-Oncologia.pdf. 
Research, Society and Development, v. 10, n. 3, e22910312657, 2021

(CC BY 4.0) | ISSN 2525-3409 | DOI: http://dx.doi.org/10.33448/rsd-v10i3.12657

Lakatos, EM; MarconI, NA. (2010) Fundamentos de Metodologia Cientifica. (7a ed.), Ed. Atlas.

Leite, M. A. C., Nogueira, D. A., \& Terra, F. D. S. (2015). Avaliação da autoestima em pacientes oncológicos submetidos a tratamento quimioterápico. Revista Latino-Americana de Enfermagem, 23(6), 1082-1089.

Lins, F. G., \& Souza, S. R. D. (2018). Formação dos enfermeiros para o cuidado em oncologia. Rev. enferm. UFPE on line, 66-74.

Macedo, A., Mercês, N. N. A. D., Silva, L. A. G. P. D., \& Sousa, G. C. C. D. (2019). Estratégias de enfrentamento dos profissionais de enfermagem frente à morte na oncologia pediátrica: revisão integrativa. Rev. pesqui. cuid. fundam. (Online), 718-724.

Minayo, M.C.S. (2013). O desafio do conhecimento: pesquisa qualitativa em saúde. (13a ed.), Hucitec.

Nascimento, A. S. M., Nobre, I. C., Lima, M. D. F. D. S., Arruda, E. F. D., \& Volpáti, N. V. (2020). Câncer infantojuvenil: Perfil dos pacientes atendidos na unidade de alta complexidade em oncologia (UNACON) em Rio Branco-Acre, Brasil, No Ano De 2017. Arq. ciências saúde UNIPAR, 35-39.

Oliveira, J., Reis, J., \& da Silva, R. (2018). Busca por cuidado oncológico: percepção de pacientes e familiares. Revista de Enfermagem UFPE on line, 12(4), 938-946.

Rodrigues, F. M., da Silva, J. K., Nunes, M. D. R., dos Santos Cardoso, L. G., \& Nascimento, L. C. (2019). Atitudes de enfermeiros na administração de quimioterápicos em oncologia pediátrica. Revista Enfermagem UERJ, 27, 37458.

Silva, L. C. A., Signor, A. C., Pilati, A. C. L., Dalfollo, B. R., \& Oliveira, D. R. (2019). Abordagem Educativa ao Paciente Oncológico: Estratégias para Orientação acerca do Tratamento Quimioterápico. Revista Brasileira De Cancerologia, 65(1), e-06305.

Silva, L. G. D., \& Moreira, M. C. (2018). Grau de complexidade dos cuidados de enfermagem: readmissões hospitalares de pessoas com câncer de mama. Revista Gaúcha de Enfermagem, 39.

Souza, N. R. D. et al. (2017). Emergência oncológica: atuação dos enfermeiros no extravasamento de drogas quimioterápicas antineoplásicas. Escola Anna Nery, 21(1).

Stübe, M., Thomé, C., Benetti, E. R. R., Gomes, J. S., \& Stumm, E. M. F. (2015). Percepções de enfermeiros e manejo da dor de pacientes oncológicos. Revista Mineira de Enfermagem, 19(3), 696-710.

Teston, E. F. et al. (2018). Sentimentos e dificuldades vivenciadas por pacientes oncológicos ao longo dos itinerários diagnóstico e terapêutico. Escola Anna Nery, 22(4).

Wakiuchi, J. et al. (2020). Sentidos e dimensões do câncer por pessoas adoecidas: análise estrutural das representações sociais. Revista da escola de enfermagem, 54(3504), 1-9. 